\title{
Contribution des Systèmes d'Information à la Performance Individuelle des Logisticiens. Le Rôle de l'Implication et de la Justice Organisationnelle
}

\author{
Arroud Maryem, Doctorante \\ Equipe de Recherche en Management \& systèmes d'information- \\ ENCG de Tanger, Maroc \\ Elkharraz Abdelilah, Enseignant-Chercheur \\ ENCG de Tanger, Responsable de l'Equipe de Recherche Management \& \\ Systèmes d'Information (ER-MSI), Tanger, Maroc \\ Boubker Omar, Enseignant-Chercheur \\ EST de Laâyoune, Université Ibn Zohr, Maroc
}

\section{Résumé}

Ce papier a pour objectif d'étudier l'impact de l'utilisation des systèmes d'information logistiques sur la performance individuelle. Pour ce faire, nous avons mobilisé une méta-analyse sur la base de trentaines d'articles provenant de dix revues prestigieuses en management des SI. Ce travail nous a permis d'identifier plusieurs indicateurs de mesure de l'impact des SI au niveau individuel. De même, il nous a permis de développer un nouveau modèle de recherche en se référant à trois champs disciplinaires, à savoir : le management des SI, le management logistique et le management des ressources humaines. À ce niveau l'adéquation tâche-technologie et la qualité perçue de la formation sont identifiées comme étant des variables qui impactent sur la perception de la qualité de l'information ainsi que la qualité technique du SILog. De même, les variables RH (l'implication et la justice organisationnelle) influencent positivement sur le niveau de la satisfaction au travail. À travers le modèle développé, la performance individuelle a été identifiée comme variable dépendante de la satisfaction au travail et de l'utilisation du SILog.

Mots clés: Systèmes d'Information Logistiques (SILog), Satisfaction, Implication, Justice Organisationnelle, Performance Individuelle 


\title{
Information Systems Contribution to Logisticians Individual Performance. The Role of Involvement and Organisational Justice
}

\author{
Arroud Maryem, Doctorante \\ Equipe de Recherche en Management \& systèmes d'information- \\ ENCG de Tanger, Maroc \\ Elkharraz Abdelilah, Enseignant-Chercheur \\ ENCG de Tanger, Responsable de l'Equipe de Recherche Management \& \\ Systèmes d'Information (ER-MSI), Tanger, Maroc \\ Boubker Omar, Enseignant-Chercheur \\ EST de Laâyoune, Université Ibn Zohr, Maroc
}

\begin{abstract}
This paper aims to study the impact of logistics information systems use on individual performance. Therefore, we have mobilised a metaanalaysis based on thirty articles coming from several well known information systems reviews. This work allowed us to identify several indicators for measuring the impact of IS at the individual level. Therefore, it has enabled us to develop a new research model by referring to three disciplinary fields, namely: Management Information Systems, Logistics Management and Human Resources Management. At this level, the task-technology suitability and perceived quality of training have been identified as variables that affect the perception of the quality of information as well as the technical quality of LIS. Similarly, HR variables (involvement and organizational justice) influence positively on the level of job satisfaction. Through the developed model, individual performance was identified as a depending variable on job'satisfaction and LIS use.
\end{abstract}

Keywords: Logistics Information Systems (LIS), Satisfaction, Involvement, Organizational Justice, Individual Performance

\section{Introduction}

Aujourd'hui, les entreprises sont confrontées à un enjeu majeur qui concerne la fidélisation de leurs salariés afin de garantir leur bien-être au travail et de s'assurer de leur forte implication organisationnelle. Du point de vue académique, la problématique d'implication au travail continue à susciter 
l'intérêt des chercheurs en Gestion des Ressources Humaines (Lacroux, 2009; Biétry \& Laroche, 2011; Mbarki et al., 2018). Cela est expliqué par le faite que l'implication constitue un facteur de performance organisationnelle et que les pratiques de gestion devraient faire en sorte de la promouvoir Thévénet (2003). Cohen (1993) a montré que l'implication organisationnelle a une influence plus forte sur l'intention de quitter l'organisation lorsque le salarié est en début de carrière. Selon Meyer, Allen et Smith (1993), les premiers mois de recrutement d'un salarié dans une organisation se comptent cruciaux pour la suite du développement du processus d'implication.

Quant aux recherches menées sur la satisfaction au travail, elles ont mis en évidence une relation inverse entre la satisfaction au travail et la rotation du personnel. C'est-à-dire, lorsque la satisfaction au travail augmente, la rotation du personnel diminue et vice versa (Hauret, 2006).

La notion de la performance de l'entreprise est multidimensionnelle. En effet, la performance organisationnelle nécessite l'intégration d'autres indicateurs à savoir ; la qualité du produit et /ou service, la mobilisation des employés, le climat de travail, la satisfaction de la clientèle. (Beaudin, Morin \& savoie ,1994).

Le cœur de notre recherche vise à présenter un modèle théorique qui permet de mesurer l'impact des systèmes d'information sur la performance individuelle des logisticiens de l'industrie automobile. Nous pensons au niveau de cette recherche que la performance individuelle et collective dépendent des éléments basiques tel que «le sens de travail » et «l'implication et la justice organisationnelle », «les attitudes» ainsi que le « comportement des individus » (Giauque, Barbey, \& Duc, 2008).

Partant de l'idée que les logisticiens (les planificateurs de production) font face à plusieurs pressions (stress) liées à la particularité de leurs tâches, qui les obligent à utiliser des systèmes d'informations logistiques (SILog) spécialisés dans le domaine.

Notre objectif est de mettre en lumière le lien existant entre l'utilisation des SILog, les pratiques Ressources Humaines et l'amélioration des conditions de travail des employés. Pour ce, on a choisi de poser notre problématique de départ de la forme suivante:

Dans quelle mesure et de quelle manière l'utilisation des Systèmes d'information logistiques contribue-elle à la performance individuelle des logisticiens?

Notre ambition s'oriente vers le développement d'un modèle permettant la configuration du rôle fondateur du département $\mathrm{RH}$ dans l'amélioration du niveau d'implication des employés utilisateurs des SI, dans le contexte de l'industrie automobile au Maroc. D'autre part, nous serons en 
mesure d'évaluer les gains apportés par une telle configuration en mettant l'être humain au centre de notre réflexion.

La recherche en management des SI s'articule autour de problématiques diverses. Plusieurs auteurs ont essayé de classifier les grandes approches de ce champ disciplinaire. Dans ce sens Rodhain et al. (2010) ont proposé d'axer les problématiques de recherche en management des systèmes d'information (MSI) sur trois niveaux: le contrôle, la stratégie et le développement.

Le management des systèmes d'information est un domaine de recherche de grande ampleur. Il s'inscrit dans une discipline qui remonte aux années 1977 (Desq et al., 2003). En effet, cette discipline a vu naissance avec l'apparition du premier numéro de la revue prestigieuse spécialisée, Management Information Systems Quarterly (MISQ). Trois ans plus tard, le premier colloque réunissant les chercheurs de la discipline avait lieu : International Conference on Information Systems (ICIS). Ce champ de recherche a fait l'objet de nombreuses analyses et études, notamment dans les deux revues académiques les plus prestigieuses spécialisées : Management Information Systems Quarterly (MISQ) et la revue Systèmes d'Information et Management (SIM).

Sur la base d'une méta-analyse de 1945 articles, couvrant 31 publications pour la période de 1977 à 2008, Rodhain et al. (2010) font la distinction entre cinq périodes d'évolution de ce champ disciplinaire.

\begin{tabular}{|c|c|l|}
\hline Périodes & Années & \multicolumn{1}{|c|}{ Caractéristiques de chaque période } \\
\hline $\begin{array}{c}\text { Le développement des } \\
\text { SI }\end{array}$ & Avant 1980 & $\begin{array}{l}\text { Recherche non empirique, sans formulation } \\
\text { d'hypothèses claires. }\end{array}$ \\
\hline La théorisation des SI & $1980-1985$ & Recherche cumulative \\
\hline La période positiviste & $1985-1990$ & $\begin{array}{l}\text { Domination des études empiriques avec } \\
\text { orientation positiviste associée à une rigueur } \\
\text { méthodologique. }\end{array}$ \\
\hline $\begin{array}{c}\text { La période de } \\
\text { diversification }\end{array}$ & $1990-2000$ & Diversification des objets de recherche \\
\hline $\begin{array}{c}\text { La période du contexte } \\
\text { social }\end{array}$ & Depuis 2000 & $\begin{array}{l}\text { Contexte social, prise en compte de l'interaction } \\
\text { entre les individus, les groupes les organisations } \\
\text { et les SI. }\end{array}$ \\
\hline
\end{tabular}

Tableau 1. Périodes de l'histoire de recherche en management des SI

À préciser que la problématique de l'évaluation des SI est identifiée comme la plus dominante (Rodhain et al. 2010 ; Petter, DeLone, \& McLean, 2012). En outre, l'être humain est considéré au centre d'évaluation des SI.

Ce travail de recherche a pour objectif de relier entre trois champs disciplinaires: les pratiques Ressources Humaines, le management des systèmes d'information et le management logistique. Pour ce faire, le présent 
papier est structuré autour de quatre points. Le premier point vise à apporter un éclairage théorique sur le management des SILog. Le deuxième point sert à entourer les soubassements théoriques de la problématique d'impact des SI au niveau individuel. Au niveau du troisième point, nous serons en mesure de présenter les différents modèles d'évaluation des SI qui constituent la base de notre modèle théorique. Le dernier point sera consacré à proposer une nouvelle configuration de l'impact des SILog sur la performance individuelle des logisticiens.

\section{Éclairage Theorique Des Concepts Cles}

Le management des SI dans l'entreprise a fait l'objet de nombreux débats scientifiques. Les chercheurs s'interrogent sur le lien existant entre l'utilisation des SILog et la performance de l'entreprise. Ainsi, le SI est au cœur de la différenciation stratégique de l'entreprise (Laudon, Laudon, Fimbel, \& Costa, 2010; Reix, Fallery, Kalika, \& Rowe, 2011). Vu la complexité de la notion de SI, la littérature en sciences de gestion nous a proposé un large panel de défintions qui ne sont pas toujours clairement établis.

\section{Essai de définition de la notion «SI »}

Mason et Mitroff (1973) sont les premiers qui ont essayé de proposer une définition à ce concept. Ils indiquent que tout SI concerne un individu, pourvu d'un profil psychologique donné, confronté à un problème décisionnel précis, dans un contexte organisationnel déterminé.

Pour d'autres chercheurs, ce domaine est construit autour de neuf environnements: le SI, l'environnement des opérations, l'environnement de développement des SI, l'environnement des utilisateurs, l'environnement de l'organisation, l'environnement externe, l'utilisation des processus métiers et le traitement des opérations (Ives, Olson, \& Baroudi, 1983).

L'auteur du modèle d'acceptation de la technologie (TAM), Davis (1989) suggère que le SI soit composé d'une infrastructure technologique, des systèmes d'application et du personnel (utilisateur des TI pour le traitement des transactions et des opérations). O’Brien (2001) souligne la notion de ressources et suggère que le SI représente un «ensemble structuré de ressources humaines, matérielles et logicielles de données et de réseaux de communication qui recueille, transforme et diffuse l'information au sein d'une entreprise ». En 2001, Davis revient pour partager la même idée, en indiquant que le SI représente l'infrastructure en technologie, les données, les applications et le personnel visant à fournir les services nécessaires en information et en communication. À préciser que la définition la plus marquante au niveau de la communauté francophone reste celle de Reix et Rowe, (2002, p. 11), qui soulignent que le concept de SI regroupe un « 
ensemble d'acteurs sociaux qui mémorisent et transforment des représentations via des technologies de l'information et des modes opératoires ». Reix, (2004, p. 3), actualise cette définition en indiquant qu'il représente: «un ensemble organisé de ressources (matériel, logiciel, personnel, données, procédures...) permettant d'acquérir, de traiter, de Stocker des informations dans et entre des organisations »(R. Reix, 2004). Morley et al., (2007) indiquent que la notion de SI renvoie à un ensemble de processus, acteurs et ressources, que l'on observe ou définit sous l'angle des informations produites et manipulées. Il peut alimenter en information d'autres systèmes de travail.

\section{Système d'information logistique et SCM}

Legrenzi (2015, p. 61) indique que le SI représente un «ensemble des ressources internes ou externes-utilisateurs, outils, données- qui contribuent au traitement (numérique ou non) de l'information». En empruntant cette définition Boubker et Chafik (2017, p. 99) suggèrent que le Système d'information logistique (SILog) « représente un ensemble de ressources en interaction, à savoir ; les ressources techniques (outils informatiques : ERP, APS, WMS, CRM, SRM), les ressources humaines (logisticiens) et l'information logistique permettant d'assurer les tâches liées aux processus logistiques de l'entreprise depuis l'amont jusqu'à l'aval de l'activité de celle$c i »$.

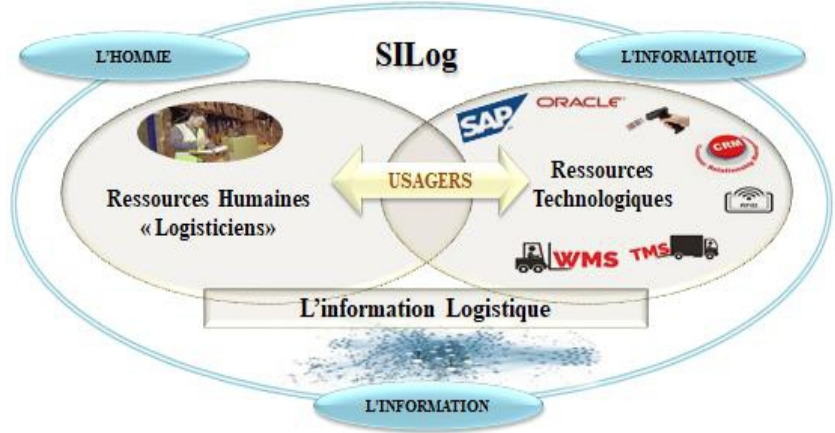

Figure 1. Composantes du SILog (Boubker et Chafik, 2017, p. 99)

Selon Livolsi et Fabbe-costes, "la relation entre logistique et SI est intime, [...] que de nombreuses entreprises ont fusionné les fonctions : le directeur logistique étant aussi directeur des SI, et investissement »(Livolsi \& Fabbe- costes, 2003, p. 2).

Dans un système où la réactivité active face aux exigences des clients constitue la clé de réussite de l'entreprise, le SILog joue un rôle primordial en permettant la synchronisation des flux logistiques tout au long de la chaine logistique (Boubker, 2015). Mentzer et al. (2001) ont défini la chaine logistique comme «un ensemble de trois ou plusieurs entités (organisations ou 
individus) directement impliquées dans les flux amont et aval de produits, services, finances, et/ou des informations d'une source à un client.»

Globalement, la littérature promeut largement l'idée que, la logistique a connu une lente maturation pour se transformer à une véritable démarche stratégique dans le fonctionnement des entreprises (Dornier\& Fender ,2007 ; Médan et al. 2008 ; Waters \& Rinsler,2014 ). Cette fonction s'est développée jusqu'à devenir une nouvelle discipline du management des entreprises (François, 2007). Saplanzani a défini le concept du Supply Chain Management (SCM) comme étant « la gestion des flux physiques et d'informations du client au fournisseur, afin d'offrir une réponse la plus satisfaisante possible aux besoins des clients. » (Saplanzani , 2003 , P. 31)

\section{« SILog » locomotive du bon fonctionnement de l'activité logistique}

Un important corpus de recherche s'intéresse à la problématique de management logistique au sein des entreprises. Comme le suggère de nombreuses définitions, la notion de la logistique renseigne le processus assurant la circulation physique des marchandises en combinant le transport, la manutention, l'emballage et toutes les opérations physiques, administratives, informationnelles et organisationnelles liées à ces mouvements (Wackermann \& Corbin, 2005). Elle met en œuvre un ensemble de méthodes pour mettre à la disposition du client la marchandise qu'il attend, au moindre coût, dans les meilleurs délais, états et quantités demandées, (Keutgen P., 2005).

Ce concept a évolué depuis, avec les évolutions des marchés et des systèmes industriels. Aujourd'hui, la fonction logistique est considérée comme stratégique pour l'entreprise. Pour Christopher (2016), cette fonction permet de maitriser les coûts et l'acquisition d'avantages concurrentiels.

La fonction logistique s'est développée jusqu'à devenir indubitablement une nouvelle discipline du management d'entreprises (François, 2007 ; Belin-Munier, 2014). En effet, elle représente une dimension critique de la performance des entreprises (Flynn, Huo, \& Zhao, 2010; Wagner, Grosse-Ruyken, \& Erhun, 2012 ; Chang et al., 2015; Boubker \& Chafik, 2015).

Dans un système où la réactivité face aux exigences des clients représente la clé de réussite, le SILog joue un rôle primordial au sein de $l^{\prime}$ entreprise en garantissant la synchronisation des flux logistiques tout au long de la Supply Chain. Leur fonctionnement est basé sur une structure à trois composantes principales (Ballou, Gilbert, \& Mukherjee, 2000): flux d'entrés, la base de données et les flux de sortie.

La coordination des flux logistiques (flux physiques: de matières premières, de produits en cours et de produits finis. flux d'informations: le niveau des stocks et des en-cours...) est capitale et conditionne le bon 
fonctionnement de la Supply Chain (Boubker \& Chafik, 2017, Boubker, 2017; Samuel \& Ruel, 2013). Ainsi, de nombreux chercheurs considèrent que le SI/TIC représente un élément crucial du bon fonctionnement de la Supply Chain, en facilitant la gestion des processus logistiques (Wood, Reiners, \& Pahl, 2015 ; 2016; Froufe, 2018). En effet, cet outil couvre l'essentiel de l'activité logistique; approvisionnement, planification de production, planification de transport, gestion de magasin/stocks, distribution ...etc (Chafik \& Boubker, 2016a,b).

Le fonctionnement de la Supply Chain est basé sur la complémentarité entre deux types d'intégration: inter-fonctionnelle et inter-organisationnelle. La première s'articule autour de l'intégration de l'ensemble des processus logistiques depuis l'amont jusqu'à l'aval (Tyndall et al. 1998). Alors que, la deuxième est construite autour d'une série de relations entre les entreprises partenaires qui partagent mutuellement les informations, les risques et les récompenses qui amènent à l'avantage compétitif (Cooper \& Ellram, 1993). Cette double intégration est assurée via l'utilisation des SILog (Boubker \& Chafik, 2016).

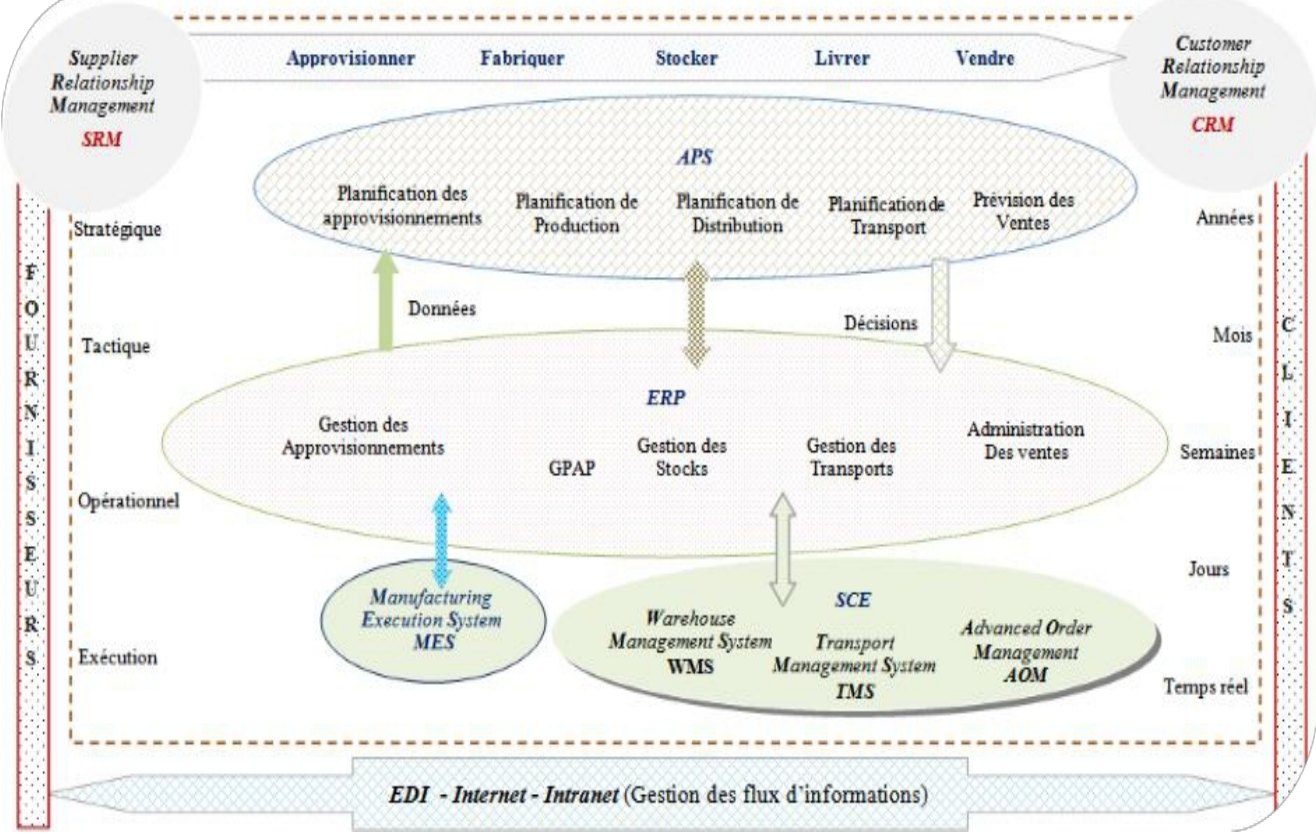

Figure 2. Couverture fonctionnelle des SILog (Boubker \& Chafik, 2016, p. 400)

\section{Soubassements Theoriques}

Cette partie vise à présenter les soubassements théoriques de la problématique d'évaluation de l'impact des SI. Ainsi, nous serons en mesure de mettre l'accent sur le niveau individuel, en précisant les principaux travaux de recherches liés à cette perspective. De même, nous synthétisons les deux 
grands courants théoriques permettant d'expliquer les modalités de la contribution des SI à la performance.

\section{Problématique d'impact des SI sur le niveau individuel}

Le management des SI a fait l'objet de nombreux débats scientifiques. Les chercheurs s'interrogent sur le lien existant entre l'utilisation des SILog et la performance de l'entreprise. Ainsi, le SI est au cœur de la différenciation stratégique de l'entreprise (Laudon et al., 2010; Robert Reix et al., 2011).

En management des SI, les chercheurs distinguent entre deux grands courants de pensée ; la perspective causale et processuel (Boubker, 2017).

La première perspective cherche à étudier la relation de cause à effet entre des variables interdépendantes tel que 1'investissement en SI et des variables dépendantes tel que la performance des individus (Missaoui, 2010). Alors que, la deuxième perspective vise à proposer une vision dynamique sur les modalités d'impact des SI sur la performance individuelle, sociale et organisationnelle.

Sur la base des travaux de Torkzadeh et Doll (1999) sur l'impact perçu des TI sur le travail des utilisateurs finaux, plusieurs dimensions ont permis de mesurer le niveau d'impact individuel (productivité et innovation dans l'exécution des tâches).

\section{Problématique de recherche}

Plusieurs chercheurs en sciences de gestion montrent que le SILog représente une composante organisationnelle facilitant l'activité de l'entreprise, via trois types de processus : transactionnels, décisionnels et de communication (Akoka \& Comyn-Wattiau, 2006). Ainsi, les SILog constituent le premier cheval de bataille pour les managers logistiques, permettant de couvrir l'essentiel de l'activité logistique (approvisionnement, planification de production, planification de transport, gestion de magasin/stocks...etc.).

Le travail de recherche de Torkzadeh et Doll (1999) qui porte sur l'impact perçu des TI sur le travail des utilisateurs finaux, a permis d'identifier plusieurs dimensions permettant de détecter ce niveau d'impact. Ainsi, la productivité des utilisateurs a été identifiée comme le premier niveau d'impact, mesurée via trois indicateurs : gain de temps, amélioration de la productivité et du travail des utilisateurs finaux.

Ainsi, nous envisageons continuer cette perspective, en essayant de proposer un modèle permettant d'évaluer la contribution de l'usage des SILog à la performance des logisticiens. En effet, nous serons en mesure d'identifier les différentes variables qui favorisent cette contribution, en se référant aux études empiriques dédiées à l'évaluation des SI (Al-Mamary et al., 2014; Almutairi \& Subramanian, 2005; DeLone \& McLean, 1992, 2003; K. C. 
Laudon \& Laudon, 2016; K. Laudon et al., 2010; Petter et al., 2008; Tam \& Oliveira, 2017), aux publications qui traitent le management des SILog (Boubker \& Chafik, 2016 ; Boubker, 2017; Samuel \& Ruel, 2013) et des travaux empiriques liés au management du capital humain (Hauret, 2006; Biétry \& Laroche, 2011; Langevin \& Mendoza, 2013; Nkakleu \& Manga, 2015; Gilbert \& Yalenios, 2017; Baggio et al., 2017). Dans ce cadre, notre problématique de recherche vise à instaurer un modèle théorique qui permet de mesurer l'utilisation des Systèmes d'information sur la performance individuelle des logisticiens.

\section{Modeles De Reference}

Après avoir présenté les grandes approches théoriques en MSI, ce deuxième axe a pour objectif d'apporter une vision critique des principaux modèles de base théoriques.

\section{Modèle de DeLone et McLean (1992)}

Ce modèle suppose que le SI est conçu avec des caractéristiques techniques (qualité du système et de l'information produite) qui influencent l'utilisation et la satisfaction de l'utilisateur final. Ainsi, ces deux variables d'attitude et de comportement conduisent à des impacts individuels qui se transforment au niveau organisationnel.

Nous avons choisi d'intégrer le modèle initial de DeLone et McLean par ce qu'il a traité l'impact de l'utilisation des SI sur la performance individuelle comme variable dépendante. Contrairement aux versions actualisées de 2003 et 2013 qui ont combinées entre la performance organisationnelle et la performance individuelle en une seule variable «Bénéfice Net ». (Petter, DeLone, \& McLean, 2013).

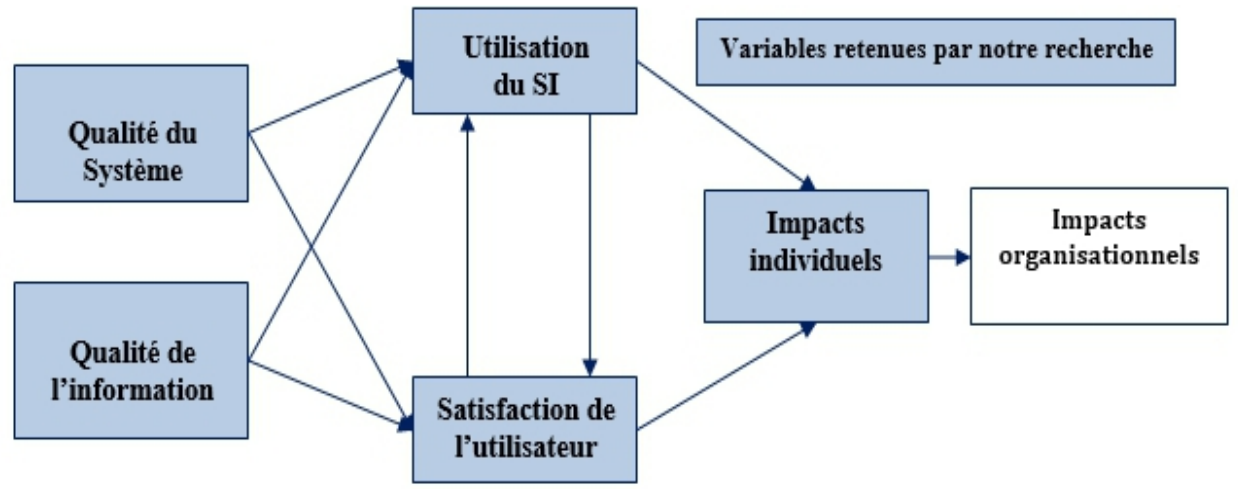

Figure 3. Modèle de Succès des SI ${ }^{5}$, (DeLone et McLean, 1992, p. 87)

${ }^{5}$ Depuis son apparition, il a été cité plus que 12. 330 fois.

Source : https://scholar.google.fr , Consulté le 11/10/2019 à 08h00. 
Modèle de Goodhue et Thompson (1995)

Ce modèle part de l'idée que l'utilisation d'un SI ne dépend pas d'une acceptation, mais plutôt, l'adéquation de la technologie avec les tâches à exécuter peut améliorer leur niveau de performance.

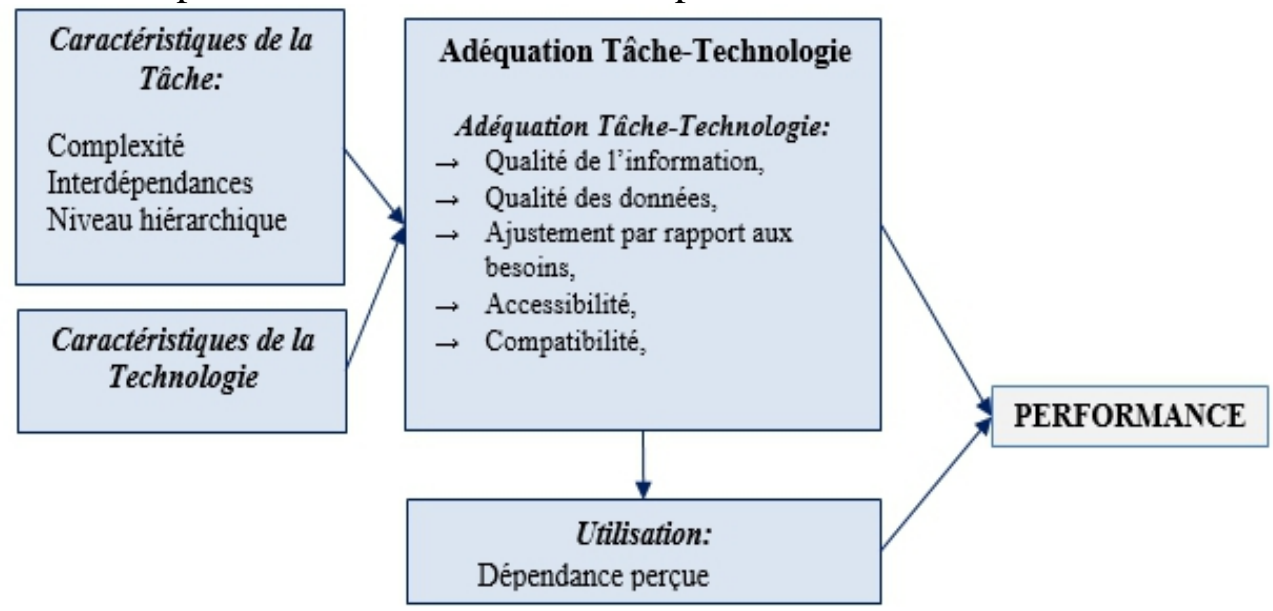

Figure 4. Le modèle d'adéquation Tâche-Technologie

(Goodhue \& Thompson, 1995, p. 225)

Tam et Oliveira (2016) proposent un nouveau modèle en reliant entre le modèle du succès de Delone et McLean ainsi que le modèle adéquation tachetechnologie (TTF) de Goodhue \& Thompson.

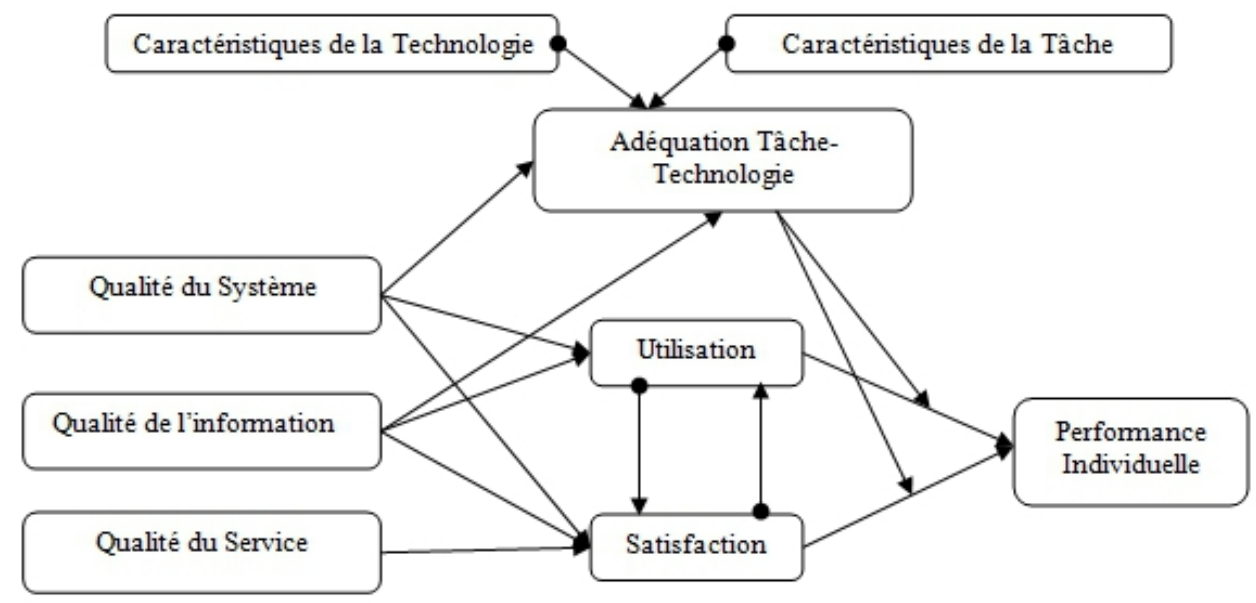

Figure 5. Modèle de l'impact des SI sur la performance individuelle des utilisateurs (Tam \& Oliveira, 2016, p. 240) 


\section{Modèle de Boubker (2017)}

Sur la base du modèle actualisé de DeLone et McLean (2003), l'extension proposée par Boubker (2017) vise à présenter un modèle permettant d'évaluer la contribution des SILog aux bénéfices logistiques.

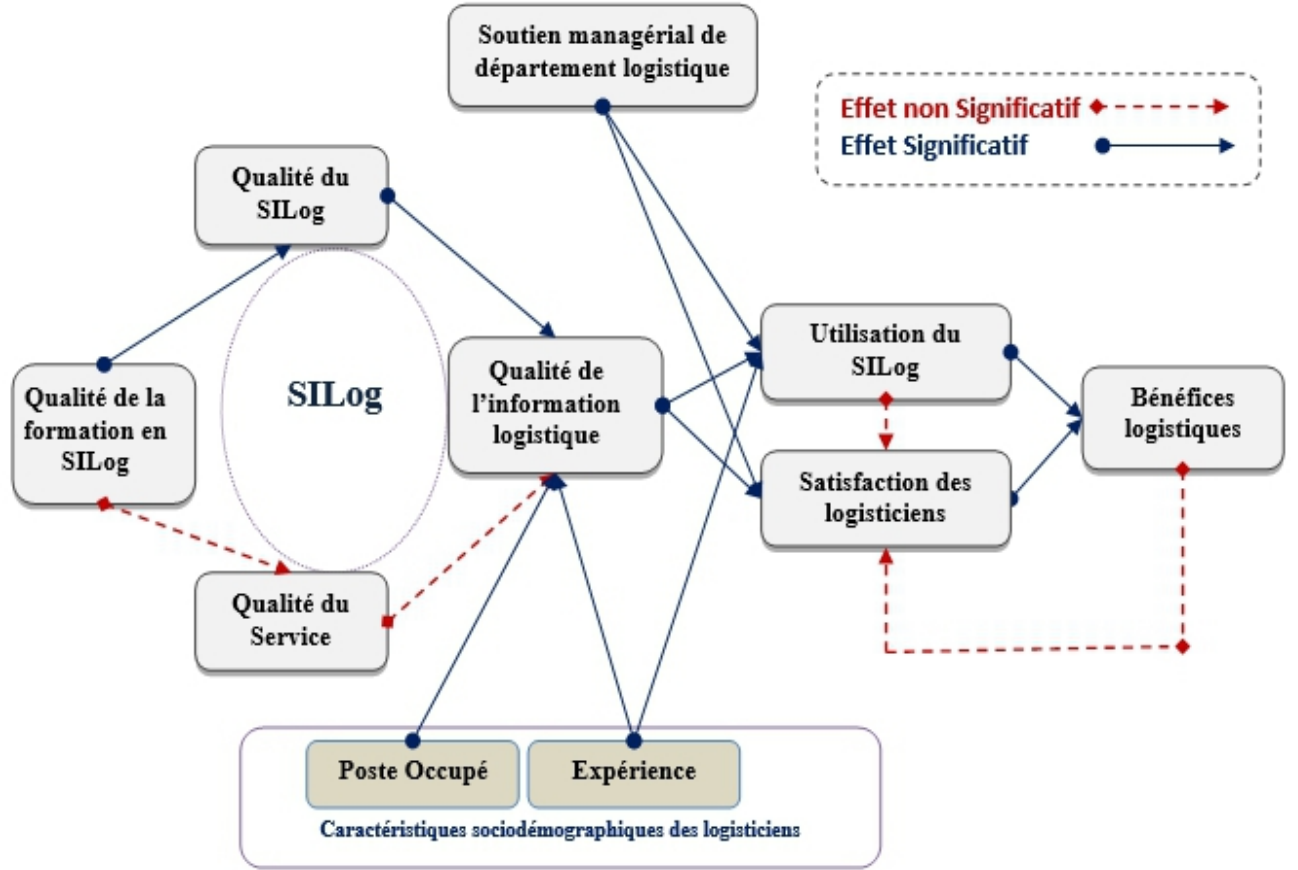

Figure 6. Modèle d'évaluation de la contribution des SILog aux bénéfices logistiques

(Boubker, 2017, p. 292)

\section{Proposition D'une Nouvelle Configuration}

Plusieurs approches théoriques coexistent pour appréhender la problématique d'évaluation des SI. Dont, les causalistes qui s'orientent vers la mesure de la relation entre l'investissement en SI et la performance de l'entreprise. Ces approches ne prennent pas en compte les acteurs ainsi elles ignorent les variables organisationnelles. Quant à notre recherche, nous allons travailler sur la problématique d'impact individuel de l'utilisation des SILog, donc la prise en compte de l'acteur (utilisateurs finaux : logisticiens) devient capitale pour aborder notre problématique.

\section{Justification du choix de niveau d'impact}

Plusieurs travaux de recherche soulignent l'importance de la prise en compte des individus utilisateurs finaux de la technologie comme point de départ pour mener à une évaluation des SI. En effet, la performance 
individuelle est le résultat d'utilisation des SI. Plusieurs recherches ont été menées pour expliquer la performance des individus au travers l'utilisation des technologies d'informations. (Tam \& Oliveira, 2017b).

Sonnentag et Frese, (2002) affirment la relation entre la performance individuelle et le bien-être au travail, pour eux la réalisation des tâches dans les meilleurs normes mène à la performance individuelle et par la suite à la satisfaction avec l'accroissement d'un sentiment de fierté. En revanche, si les tâches ne sont pas complètement achevées, ceci provoque une situation d'échec qui se traduit par l'insatisfaction des individus.

\section{Approche théorique mobilisée : la théorie de la psychologie sociale}

La psychologie sociale met au cœur de sa réflexion la notion $\mathrm{du}$ groupe, elle s'intéresse particulièrement à l'étude des groupes restreints, c'està-dire des groupes composés de trois à vingt personnes. (Bertrand, 2013). Un groupe se définit comme «un ensemble de personnes ayant la possibilité de se percevoir et d'interagir directement en participant à une activité commune grâce à un système de règles et de normes formelles ou informelles. » (Abric, 2004)

Afin de définir un groupe, certaines caractéristiques sont nécessaires :

- L'existence de relations interpersonnelles entre les membres générant un système d'influences réciproques ;

- La poursuite d'un but commun ;

- La présence d'une organisation dans laquelle le rôle de chaque membre sera plus ou moins défini.

Elton Mayo (1930) a mené une expérimentation avec son équipe dans les ateliers Hawthorne de la Western Electric à partir de 1933, leurs résultats montrent que la performance individuelle dépend des relations interpersonnelles qui sont tissées entre les membres du groupe et cela quel que soit leur statut hiérarchique (Bertrand, 2013).

Lewin (1939) a constaté lors de ses recherches sur le changement des groupes, que ces derniers sont plus accessibles au changement d'opinions ou d'attitudes qu'un individu isolé. C'est le travail groupal qui permet d'instaurer les normes communes et de construire de nouvelles normes qui sont à l'origine du changement d'attitudes.

Notre champ d'étude au niveau de ce travail de recherche, s'intéresse à l'étude de l'impact des technologies d'information sur la performance des groupes d'individus au sein de l'industrie du câblage automobile.

De nombreux travaux empiriques se sont intéressés à l'étude de l'impact des technologies sur la performance des entreprises, à travers leurs impacts intermédiaires sur les individus et/ou les groupes d'individus (Zmud, 
1979; Azjen et Fishbein, 1980; Ives et Olson, 1984 ; Davis, 1989 ; DeLone et McLean, 1992 ; DeLone et McLean, 2003 ; Petter, DeLone, \& McLean, 2012).

Afin de choisir l'approche théorique en adéquation avec notre recherche, nous envisageons se concentrer sur les approches processuelles qui placent l'être humain au centre de leur réflexion. Dans ce sens, l'approche d'évaluation fondée sur la psychologie sociale met l'accent sur les modalités d'interaction entre la technologie et les individus (à travers les facteurs psychosociaux: attitudes, croyances et comportements).
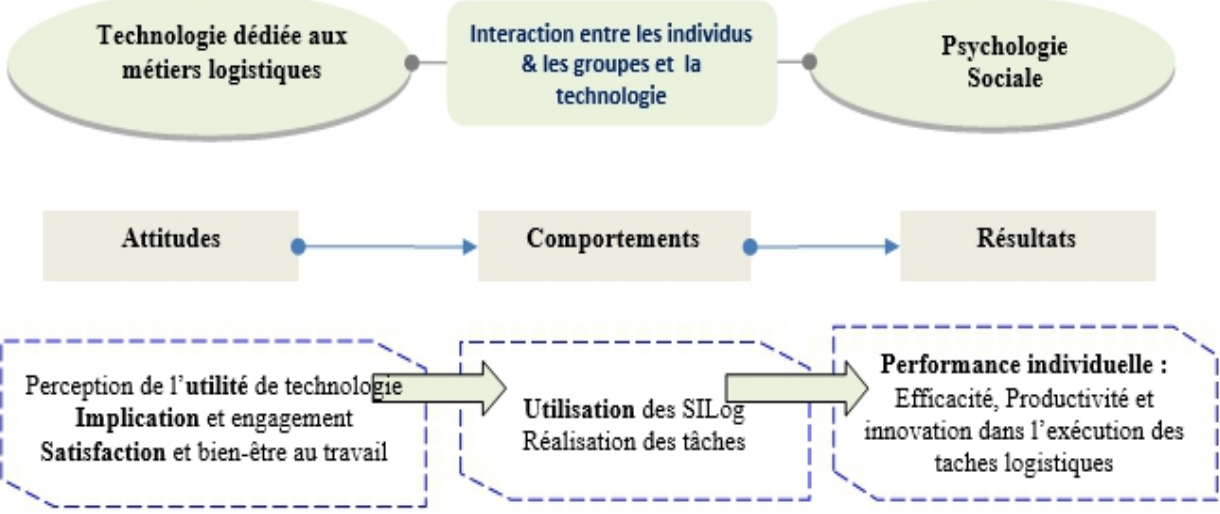

Figure 7. SILog et performance individuelle des logisticiens à l'optique de la théorie de la psychologie sociale

En se basant sur la théorie de la psychologie sociale, nous envisageons adopter le modèle de Boubker (2017) qui traite la problématique de la contribution des SILog aux bénéfices logistiques des entreprises de l'industrie automobile et aéronautique au Maroc, comme modèle de base théorique. L'objectif est de procéder à son extension en ajoutant des variables RH liées au contexte marocain.

\section{Essai d'élaboration d'une nouvelle configuration de l'étude de l'impact des SILog sur le niveau individuel}

L'originalité de notre recherche consiste à marier entre trois disciplines scientifiques, à savoir : le management logistique, le management des SI et le management des ressources humaines. Boubker (2017, P. 310-311) suggèrent qu' "afin de proposer un modèle plus complexe avec un pouvoir prédictif élevé, il est intéressant d'introduire d'autres variables au modèle développé et testé. En premier lieu, la justice au travail (procédurale, distributive et organisationnelle), le bien-être psychologique et l'implication des logisticiens peuvent être une piste envisageable entant que variables liées au Management des $R H »$.

En suivant cette perspective, nous choisissons d'intégrer des variables liées au management des ressources humaines afin d'évaluer la contribution des SILog à la performance individuelle des logisticiens. 


\begin{tabular}{|c|c|c|}
\hline Hypothèses & Auteurs & Type de recherche \\
\hline \multirow{3}{*}{$\begin{array}{l}\text { H } 1: \text { La perception de la qualité de } \\
\text { formation a un effet positif sur la } \\
\text { perception de la qualité du SILog. }\end{array}$} & (Sabherwal et al.,2006) & Méta-Analyse \\
\hline & (Bradley et al., 2006) & Test Empirique \\
\hline & (Boubker, 2017) & Test Empirique \\
\hline \multirow{2}{*}{$\begin{array}{l}\text { H } 2 \text { : La perception de la qualité de } \\
\text { formation a un effet positif sur la } \\
\text { perception de la qualité des } \\
\text { informations }\end{array}$} & (Sabherwal et al., 2006) & Test Empirique \\
\hline & (Bradley et al., 2006) & Test Empirique \\
\hline \multirow{2}{*}{$\begin{array}{l}\text { H } 3 \text { : L'adéquation Tache- } \\
\text { Technologie a un effet positif sur la } \\
\text { perception de la qualité du SILog. }\end{array}$} & (Goodhue \& Thompson, 1995) & Test Empirique \\
\hline & (Tam \& Oliveira, 2016) & Test Empirique \\
\hline \multirow{2}{*}{$\begin{array}{l}\text { H } 4: \text { TTF a un effet positif sur la } \\
\text { perception de la qualité des } \\
\text { informations }\end{array}$} & (Goodhue \& Thompson, 1995) & Test Empirique \\
\hline & (Tam \& Oliveira, 2016) & Test Empirique \\
\hline \multirow{4}{*}{$\begin{array}{l}\text { H } 5 \text { : La perception de la qualité } \\
\text { technique du SILog a un effet } \\
\text { positif sur la perception de la qualité } \\
\text { des informations logistiques. }\end{array}$} & (Gorla, Somers, \& Wong, 2010) & Test Empirique \\
\hline & (Hasan et al., 2013) & État de l'Art \\
\hline & (Al-Mamary et al., 2014) & État de l'Art \\
\hline & (Boubker, 2017) & Test Empirique \\
\hline \multirow{5}{*}{$\begin{array}{l}\text { H 6: La perception de la qualité des } \\
\text { informations logistiques a un effet } \\
\text { positif sur l'utilisation du SILog. }\end{array}$} & (Rai, Lang, \& Welker, 2002) & Test Empirique \\
\hline & (DeLone \& McLean, 2003) & État de l'Art \\
\hline & (Urbach et al., 2008) & État de l'Art \\
\hline & (Petter \& McLean, 2009) & Méta-Analyse \\
\hline & (Boubker \& Chafik, 2017) & Test Empirique \\
\hline \multirow{6}{*}{$\begin{array}{l}\text { H 7: La perception de la qualité des } \\
\text { informations logistiques a un effet } \\
\text { positif sur la satisfaction des } \\
\text { logisticiens. }\end{array}$} & (DeLone \& McLean, $1992 ; 2003$ ) & État de l'Art \\
\hline & (Sørum et al., 2012) & Test Empirique \\
\hline & (Baudet, 2015) & Test Empirique \\
\hline & (Yatin et al., 2015) & Test Empirique \\
\hline & (Ghasemaghaei \& Hassanein, 2016) & Test Empirique \\
\hline & (Boubker, 2017) & État de l'Art \\
\hline \multirow{3}{*}{$\begin{array}{l}\text { H } 8 \text { : Le soutien managérial } \\
\text { logistique a un effet positif sur } \\
\text { l'utilisation du SILog. }\end{array}$} & (Venkatesh et al., 2003) & Méta-Analyse \\
\hline & (Petter \& McLean, 2009) & Test Empirique \\
\hline & (Boubker, 2017) & Méta-Analyse \\
\hline \multirow{4}{*}{$\begin{array}{l}\text { H } 9 \text { : Le soutien managérial } \\
\text { logistique a un effet positif sur la } \\
\text { satisfaction et bien-être au travail } \\
\text { des logisticiens. }\end{array}$} & (Urbach et al . 2010) & Test Empirique \\
\hline & (Petter et al., 2012) & Méta-Analyse \\
\hline & Al-Mamary et al. (2015) & Test Empirique \\
\hline & (Boubker, 2017) & Test Empirique \\
\hline \multirow{7}{*}{$\begin{array}{l}\text { H } 10 \text { : La justice organisationnelle a } \\
\text { un effet positif sur le niveau de } \\
\text { satisfaction et bien-être au travail } \\
\text { des logisticiens. }\end{array}$} & (Alexander \& Ruderman, 1987) & Test Empirique \\
\hline & (Masterson, et al., 2000) & Test Empirique \\
\hline & (Cohen-Charash \& Spector, 2001) & Méta-analyse \\
\hline & (Cropanzano et al., 2002) & État de l'Art \\
\hline & (Müller \& Djuatio, 2013) & Test Empirique \\
\hline & (Akremi et al., 2006) & État de l'Art \\
\hline & (Müller \& Djuatio, 2011) & Test Empirique \\
\hline \multirow{5}{*}{$\begin{array}{l}\text { H } 11 \text { : L'implication des logisticiens } \\
\text { a un effet positif sur leur niveau de } \\
\text { satisfaction et bien-être au travail. }\end{array}$} & (Aranya et al., 1982) & État de l'art \\
\hline & (Bateman \& Strasser, 1984) & Méta-analyse \\
\hline & (Hauret, 2006) & Test Empirique \\
\hline & (DeLone \& McLean, 1992) & Méta-analyse \\
\hline & (DeLone \& McLean, 2004) & Test Empirique \\
\hline
\end{tabular}




\begin{tabular}{|c|c|c|}
\hline \multirow{2}{*}{$\begin{array}{l}\text { H } 12 \text { : L'utilisation des SILog a un } \\
\text { effet positif sur le niveau de } \\
\text { performance individuelle. }\end{array}$} & (Tam \& Oliveira, 2016) & Test Empirique \\
\hline & (Tam \& Oliveira, 2017) & Test Empirique \\
\hline \multirow{4}{*}{$\begin{array}{l}\text { H } 13 \text { : La satisfaction des } \\
\text { logisticiens a un effet positif sur le } \\
\text { niveau de performance individuelle. }\end{array}$} & (DeLone \& McLean, 1992) & Test Empirique \\
\hline & (DeLone \& McLean, 2004) & Test Empirique \\
\hline & (Tam \& Oliveira, 2016) & Test Empirique \\
\hline & (Tam \& Oliveira, 2017) & Test Empirique \\
\hline
\end{tabular}

Tableau 2. Justification du choix des hypothèses

En se basant sur le modèle initial de DeLone et McLean (1992), le modèle de Goodhue et Thompson (1995), le modèle de Tam et Oliveira (2016), le modèle d'implication organisationnelle et la satisfaction au travail (Hauret, 2006), ainsi que le modèle de Boubker (2017) nous pouvons ainsi, proposer un modèle de recherche théorique.

Le modèle développé via la prise en compte d'une perspective $\mathrm{RH}$, vise à évaluer la performance individuelle des logisticiens vis-à-vis de l'utilisation des SI, on a pris en considération les variables liées à la qualité de système et de l'information, ces dernières ont un impact sur la satisfaction au travail.

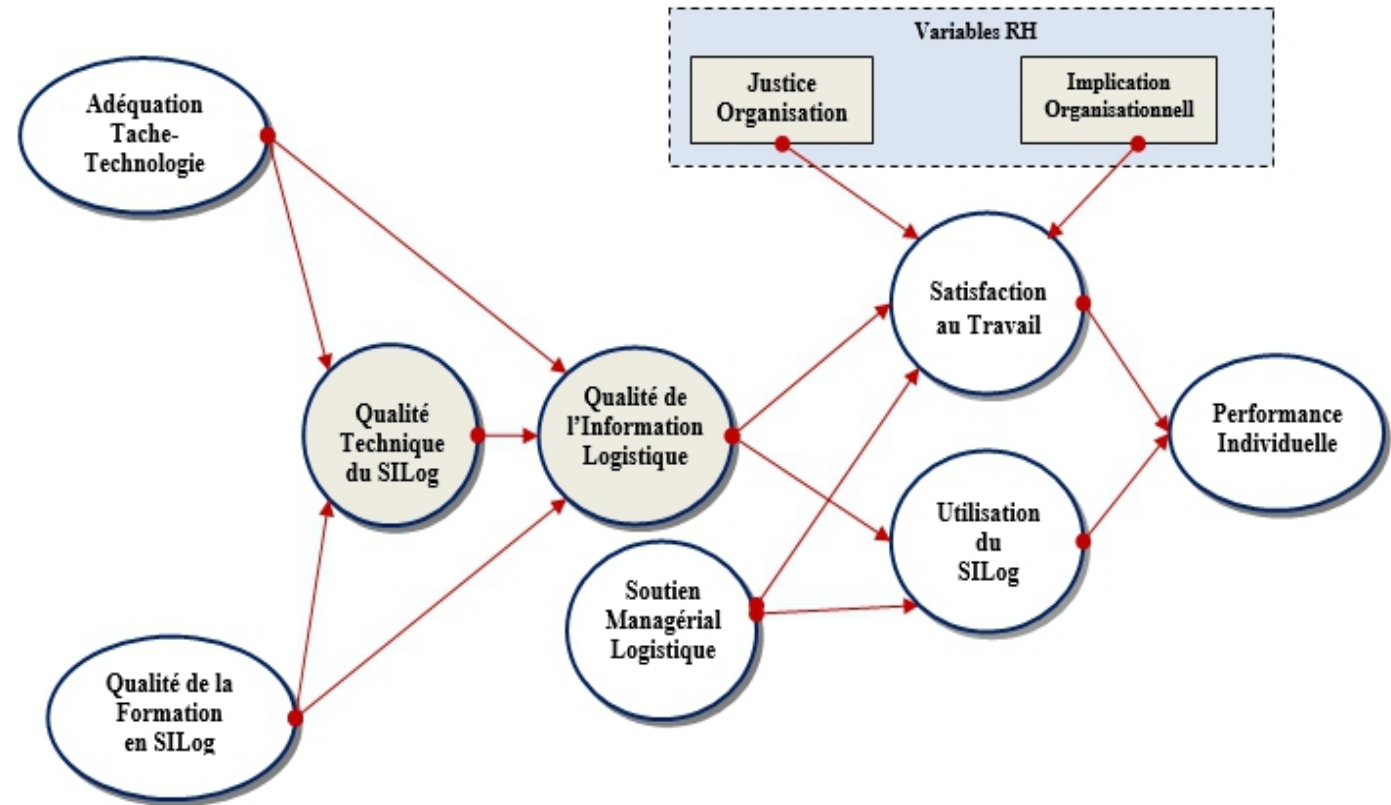

Figure 8. Modèle d'évaluation de la contribution des SILog à la performance individuelle

(Source : notre propre élaboration)

Le tableau ci-dessous synthétise les différentes définitions des variables retenues au niveau du modèle de recherche. 


\begin{tabular}{|c|c|}
\hline Variables & Définitions et auteurs \\
\hline La formation & $\begin{array}{l}\text { "Les efforts fournis afin de transmettre un savoir relatif aux SI». } \\
\text { (Ryan Nelson \& Cheney, 1987, p.548) }\end{array}$ \\
\hline $\begin{array}{l}\text { Qualité technique } \\
\text { du SILog }\end{array}$ & $\begin{array}{l}\text { "La croyance des logisticiens utilisateurs relative à la qualité } \\
\text { technique du SI » (Nelson, Todd, \& Wixom, 2005) }\end{array}$ \\
\hline $\begin{array}{l}\text { Qualité de } \\
\text { l'information }\end{array}$ & $\begin{array}{l}\text { «Les caractéristiques souhaitables des Sorties du système» }{ }^{6} \text { (Petter } \\
\text { et al., } 2008, \text { p. } 239)\end{array}$ \\
\hline $\begin{array}{l}\text { Utilisation du } \\
\quad \text { SILog }\end{array}$ & $\begin{array}{l}\text { «Le degré et la manière dont le personnel et les clients utilisent les } \\
\text { capacités d'un système d'information» }{ }^{7} \text { (Petter et al., 2008, p. 239) }\end{array}$ \\
\hline $\begin{array}{l}\text { Satisfaction au } \\
\text { travail }\end{array}$ & $\begin{array}{l}\text { "Résulte de l'adéquation entre les perceptions que la personne a des } \\
\text { différents aspects de son emploi et les perceptions qu'elle a quant à } \\
\text { ce que devraient être les différentes facettes de son travail. La } \\
\text { satisfaction dépend du niveau de divergence entre ce que la personne } \\
\text { désire et ce qu'elle en retire », (Hauret, } 2006, p .128)\end{array}$ \\
\hline $\begin{array}{c}\text { Justice } \\
\text { organisationnelle }\end{array}$ & $\begin{array}{l}\text { La justice organisationnelle s'intéresse aux perceptions véhiculées } \\
\text { par les membres d'une organisation quant à la manière plus ou } \\
\text { moins juste dont ils sont traités (Langevin \& Mendoza, 2013, p. 34). } \\
\text { Dimensions de la justice: distributive; procédurale et } \\
\text { interactionnelle. }\end{array}$ \\
\hline $\begin{array}{l}\text { Implication } \\
\text { organisationnelle }\end{array}$ & $\begin{array}{l}\text { "l'ensemble des prédispositions mentales ou un état psychologique } \\
\text { (sentiments et/ou croyances) concernant la relation d'un employé } \\
\text { avec l'organisation », (Allen \& Meyer, 1990; cité par Mhiri, 2019, } \\
\text { p. 38). Dimensions d'implication : Affective, Calculée et } \\
\text { Normative (Allen \& Meyer, 1990; Meyer \& Allen, 1991). }\end{array}$ \\
\hline $\begin{array}{c}\text { Performance } \\
\text { individuelle } \\
\text { au travail }\end{array}$ & $\begin{array}{l}\text { "La performance au travail est définie comme la valeur totale } \\
\text { attendue par l'organisation des épisodes de comportements discrets } \\
\text { qu'exerce un individu pendant une période de temps donnée » } \\
\text { Motowidlo }(2003, \mathrm{p} .39)^{8} \text {. Elle renvoie à la performance dans la tâche } \\
\text { logistique et la performance contextuelle. }\end{array}$ \\
\hline
\end{tabular}

Tableau 3. Définitions des différentes variables retenues

\section{Conclusion}

Ce papier avait pour objectif de proposer un nouveau modèle théorique permettant de mettre en lumière les modalités de la contribution des SILog à la performance individuelle des logisticiens.

Pour ce faire, nous avons présenté dans un premier point les soubassements théoriques liées à la problématique d'évaluation des SI, en mettant l'accent sur le niveau individuel. Ce choix a été justifié par la mobilisation de la théorie de la psychologie sociale qui s'intéresse à l'étude des comportements des groupes d'individus et les relations interpersonnelles qui se nouent entre eux quel que soit leurs statuts hiérarchiques.

6 « The desirable characteristics of the system outputs »

${ }^{7}$ "The degree and manner in which staff and customers utilize the capabilities of an information system »

${ }^{8}$ «Job performance is defined as the total expected value to the organization of the discrete behavioral episodes that an individual carries out over a standard period of time " 
Par la suite, nous avons présenté les principaux modèles de références théoriques. En troisième lieu, nous avons justifié la théorie de la psychologie sociale comme une perspective théorique en étroite adéquation avec notre problématique de recherche. Enfin, nous avons mis l'accent sur le modèle de base, ainsi que les variables d'extension repérées et intégrées dans le nouveau modèle.

Notre recherche comporte plusieurs apports théoriques. En premier lieu, elle souligne l'importance d'orienter l'évaluation des SI vers le niveau individuel. De même, elle avance la centralité de la prise en compte de l'adéquation des tâches logistiques avec la technologie utilisée. En dernier lieu, un apport majeur provient de la mobilisation d'une perspective axée sur le management des ressources humaines.

Nous envisageons continuer notre champ d'investigation en optant pour une approche hypothético-déductive basée sur une analyse factorielle exploratoire (AFE), puis confirmatoire (AFC) sous l'approche PLS, auprès des logisticiens des entreprises de l'industrie automobile au Maroc (Thiétart et al., 2014).

\section{References:}

1. Akremi, A. E., Guerrero, S., \& Neveu, J.-P. (2006). Comportement organisationnel-Vol. 2: Justice organisationnelle, enjeux de carrière et épuisement professionnel. De Boeck Supérieur.

2. Alexander, S., \& Ruderman, M. (1987). The role of procedural and distributive justice in organizational behavior. Social Justice Research, 1(2), 177-198.

3. Allen, N. J., \& Meyer, J. P. (1990). The measurement and antecedents of affective, continuance and normative commitment to the organization. Journal of Occupational and Organizational Psychology, 63(1), 1-18.

4. Al-Mamary, Y. H., Shamsuddin, A., \& Nor Aziati, A. H. (2014). The Relationship between System Quality, Information Quality, and Organizational Performance. International Journal of Knowledge and Research in Management \& E-Commerce, 4(3), 07-10.

5. Almutairi, H., \& Subramanian, G. H. (2005). An empirical application of the DeLone and McLean model in the Kuwaiti private sector. Journal of Computer Information Systems, 45(3), 113-122.

6. Aranya, N., Lachman, R., \& Amernic, J. (1982). Accountants' job satisfaction: A path analysis. Accounting, Organizations and Society, 7(3), 201-215.

7. Baggio, S., Dufour, F., \& Sutter, P.-E. (2017). Mesurer le risque social en entreprise. Le modèle de la réluctance socio-organisationnelle. Psychologie Du Travail et Des Organisations, 23(1), 14-28. 
8. Ballou, R. H., Gilbert, S. M., \& Mukherjee, A. (2000). New managerial challenges from supply chain opportunities. Industrial Marketing Management, 29(1), 7-18.

9. Bateman, T. S., \& Strasser, S. (1984). A longitudinal analysis of the antecedents of organizational commitment. Academy of Management Journal, 27(1), 95-112.

10. Baudet, C. (2015). L'influence de la participation des utilisateurs sur le succès des systèmes d'information. Business Science Institute; Université Lyon III, Institut d'Administration des Entreprises.

11. Belin-Munier, C. (2014). Logistique, chaîne logistique et SCM dans les revues francophones de gestion : quelle dimension stratégique? In XXIIIème conférence de l'Association Internationale de Management Stratégique (AIMS) (p. 26-p).

12. Biétry, F., \& Laroche, P. (2011). L'implication dans l'organisation, le syndicat et/ou la carrière, Organizational commitment, union commitment and/or career commitment. Revue de gestion des ressources humaines, (81), 19-38.

13. Boubker, O. (2015). Contribution des TIC à la performance de la logistique de distribution. Éditions Universitaires Européennes. ISBN : 978-3-8416-6010-7.

14. Boubker O., Chafik K. (2015). Élaboration d'un modèle théorique d'évaluation de la contribution des SIL à la performance et la maturité des processus logistiques ». Proceedings of the $X^{\text {th }}$ International Conference on Integrated Design and Production, Dec 2015, TangierMorocco. Submitted on 22 Jan 2016, <hal-01260767>. Accessible en ligne sur : https://hal.archives-ouvertes.fr/hal-01260767v1.

15. Boubker, O., \& Chafik, K. (2016). Comprendre le rôle des systèmes d'information dans l'amélioration de la performance et la maturité des processus logistiques : étude qualitative auprès de six entreprises de l'industrie automobile et aéronautique au Maroc. European Scientific Journal, ESJ, 12(28), 397-429. doi: 10.19044/esj.2016.v12n28p397

16. Boubker, O., \& Chafik, K. (2017). An investigation of the relationship between logistics information quality and LIS use based on structural equation modelling approach: A case study of automotive and aerospace companies in Morocco. New Trends and Issues Proceedings on Humanities and Social Sciences., 4(10), 97-109. DOI: 10.18844/prosoc.v4i10.3068

17. Boubker, O. (2017). Évaluation de la contribution des systèmes d'information à la performance et à la maturité des processus logistiques : Cas des entreprises de l'industrie automobile et aéronautique au Maroc. (Thèse de doctorat en Sciences de Gestion. Université Abdelmalek Essaadi, ENCG de Tanger). 
18. Bradley, R. V., Pridmore, J. L., \& Byrd, T. A. (2006). Information systems success in the context of different corporate cultural types: an empirical investigation. Journal of Management Information Systems, 23(2), 267-294.

19. Chafik, K., Boubker, O. (2016a), « Systèmes d'information et pratiques logistiques : analyse basée sur le modèle SCOR. Cas d'une entreprise de l'industrie automobile marocaine ». Revue Marocaine de Management Logistique et Transport, 1(1), p. 45-74.

20. Chafik, K., \& Boubker, O. (2016b). Systèmes d'information, performance et maturité des processus : Élaboration d'un modèle théorique basé sur l'approche sociotechnique. International Journal of Innovation and Applied Studies, 16(1), 111-126.

21. Chang, W., Ellinger, A. E., Kim, K. K., \& Franke, G. R. (2015). Supply chain integration and firm financial performance: A meta-analysis of positional advantage mediation and moderating factors. European Management Journal, 34(3), 282-295.

22. Christopher, M. (2016). Logistics \& supply chain management. Pearson UK.

23. Cohen-Charash, Y., \& Spector, P. E. (2001). The Role of Justice in Organizations: A Meta-Analysis. Organizational Behavior and Human Decision Processes, 86(2), 278-321.

24. Cooper, M. C., \& Ellram, L. M. (1993). Characteristics of Supply Chain Management and the Implications for Purchasing and Logistics Strategy. Int Jrnl Logistics Management, 4(2), 13-24.

25. Cropanzano, R., Prehar, C. A., \& Chen, P. Y. (2002). Using social exchange theory to distinguish procedural from interactional justice. Group \& Organization Management, 27(3), 324-351.

26. Davis, F. D. (1989). Perceived usefulness, perceived ease of use, and user acceptance of information technology. MIS Quarterly, 13(3), 319-340.

27. DeLone, W. H., \& McLean, E. R. (1992). Information systems success: The quest for the dependent variable. Information Systems Research, 3(1), 60-95.

28. DeLone, W. H., \& McLean, E. R. (2003). The DeLone and McLean Model of Information Systems Success: A Ten-Year Update. Journal of Management Information Systems, 19(4), 9-30.

29. DeLone, W. H., \& McLean, E. R. (2004). Measuring e-Commerce Success: Applying the DeLone \& McLean Information Systems Success Model. International Journal of Electronic Commerce, 9(1), $31-47$. 
30. Desq, S., Fallery, B., Reix, R., \& Rodhain, F. (2003). 25 ans de recherche en Systèmes d'Information. Systèmes d'information et Management, 7(3), 5-31.

31. Giauque, D., Barbey, V., \& Duc, N. (2008). Les leviers de la performance individuelle et collective dans les organisations publiques Suisses: l'importance d'un pilotage participatif. Revue française d'administration publique, 128(4), 785.

32. Flynn, B. B., Huo, B., \& Zhao, X. (2010). The impact of supply chain integration on performance: A contingency and configuration approach. Journal of Operations Management, 28(1), 58-71.

33. François. (2007). Planification des chaînes logistiques : Modélisation du système décisionnel et performance. (Thèse en Productique). Bordeaux: Université Bordeaux I.

34. Froufe, S. (2018). Une caractérisation de la contribution des logisticiens à la mise en cohérence opérationnelle des systèmes d'information et de la logistique : le cas de l'aéronautique. Logistique \& Management, 26(1), 50-57.

35. Ghasemaghaei, M., \& Hassanein, K. (2016). A macro model of online information quality perceptions: A review and synthesis of the literature. Computers in Human Behavior, 55, 972-991.

36. Gilbert, P., \& Yalenios, J. (2017). Conclusion. Repères, 109-110.

37. Goodhue, D. L., \& Thompson, R. L. (1995). Task-technology fit and individual performance. MIS Quarterly, 19(2), 213-236.

38. Gorla, N., Somers, T. M., \& Wong, B. (2010). Organizational impact of system quality, information quality, and service quality. The Journal of Strategic Information Systems, 19(3), 207-228. https://doi.org/10.1016/j.jsis.2010.05.001

39. Hasan, Y., Shamsuddin, A., \& Aziati, N. (2013). The impact of management information systems adoption in managerial decision making: A review. The International Scientific Journal of Management Information Systems, 8(4), 010-017.

40. Hauret, C. P. dit. (2006). L'implication organisationnelle et la satisfaction au travail sont-ils des concepts pertinents pour les expertscomptables stagiaires ? Comptabilité - Contrôle - Audit, Tome 12(1), 125-146. https://doi.org/10.3917/cca.121.0125

41. Ibn El Farouk., Jawab, F., (2017, April). Synchronous flow in automotive industry: Case study of RENAULT. In Logistics and Supply Chain Management (LOGISTIQUA), 2017 International Colloquium on (pp. 199-203).

42. Ives, B., Olson, M. H., \& Baroudi, J. J. (1983). The measurement of user information satisfaction. Communications of the ACM, 26(10), 785-793. 
43. Keutgen P. (2005). Les parcs logistiques en Europe du Nord-Ouest (Mémoire de fin d'études en Sciences géographiques). Université de Liège.

44. Lacroux, A. (2009). L’analyse des modèles de relations structurelles par la méthode PLS: une approche émergente dans la recherche quantitative en GRH. In XXème congrès de l'AGRH, Toulouse du (Vol. 9).

45. Langevin, P., \& Mendoza, C. (2013). La Justice : un revenant au pays du Contrôle?, Justice : A Ghost Back in the Management Control Area ? Comptabilité - Contrôle - Audit, Tome 19(1), 33-57.

46. Laudon, K. C., \& Laudon, J. P. (2016). Management information system. Pearson Education India.

47. Laudon, K., Laudon, J., Fimbel, É., \& Costa, S. (2010). Management des systèmes d'information. Pearson Education France.

48. Legrenzi, C. (2015). Informatique, numérique et système d'information: définitions, périmètres, enjeux économiques. Vie \& sciences de l'entreprise, 2(200), 49-76.

49. Mason, R. O., \& Mitroff, I. I. (1973). A Program for Research on Management Information Systems. Management Science, 19(5), 475487.

50. Masterson, S. S., Lewis, K., Goldman, B. M., \& Taylor, M. S. (2000). Integrating justice and social exchange: The differing effects of fair procedures and treatment on work relationships. Academy of Management Journal, 43(4), 738-748.

51. Mbarki M.A. Rharzouz J., Boubker, O. (2018). l'impact de la formation, selon les normes qualité, sur l'implication du personnel sous contrats flexibles : résultats d'une étude empirique ». European Scientific Journal, 4 (2), p. 173-87. Doi: 10.19044/esj.2018.v14n2p173

52. Meyer, J. P., \& Allen, N. J. (1991). A three-component conceptualization of organizational commitment. Human Resource Management Review, 1(1), 61-89.

53. Missaoui, I. (2010). Valeur et performance des SI (Cahier de recherche n'5). CIGREF.

54. Mhiri, S. (2019). Les effets de la charge de travail et du plafonnement de carrière sur les trois dimensions de l'implication organisationnelle des cadres. Revue de gestion des ressources humaines, (1), 35-55.

55. Motowidlo, S.J. (2003), Job performance, dans Handbook of Psychology, Industrial and Organizational Psychology, sous la direction de W.C Borman, D.R. Ilgen, R.J. Klimoski et I.B. Weiner, Hoboken, NJ: Wiley, vol. 12, pp. 39-52. 
56. Müller, J., \& Djuatio, E. (2011). Les relations entre la justice organisationnelle, l'employabilité, la satisfaction et l'engagement organisationnel des salariés, The relationships between organizational justice, employability job satisfaction and organizational commitment of employees. Revue de gestion des ressources humaines, 4(82), 4662.

57. Müller, J., \& Djuatio, E. (2013). Les relations entre la justice organisationnelle, l'employabilité, la satisfaction et l'engagement organisationnel des salariés, The relationships between organizational justice, employability job satisfaction and organizational commitment of employees. Revue de gestion des ressources humaines, (82), 46-62.

58. Nelson, R. R., \& Cheney, P. H. (1987). Training end users: an exploratory study. MIS quarterly, 547-559.

59. Nelson, R. R., Todd, P. A., \& Wixom, B. H. (2005). Antecedents of information and system quality: an empirical examination within the context of data warehousing. Journal of management information systems, 21(4), 199-235.

60. Nkakleu, R., \& Manga, B. (2015). L'implication organisationnelle des personnels-cadres dans les PME : le rôle de la tontine d'entreprise en contexte camerounais1. Revue internationale P.M.E., 28(3-4), 287313.

61. O'BRIEN, J. A. (2001). Introduction aux systèmes d'information: un outil essentiel pour l'entreprise branchée, Chenelière. McGraw-Hill, Montréal.

62. Petter, S., DeLone, W., \& McLean, E. (2008). Measuring information systems success: models, dimensions, measures, and interrelationships. Eur J Inf Syst, 17(3), 236-263.

63. Petter, S., DeLone, W., \& McLean, E. R. (2012). The past, present, and future of" IS Success". Journal of the Association for Information Systems, 13(5), 341.

64. Petter, S., \& McLean, E. R. (2009). A meta-analytic assessment of the DeLone and McLean IS success model: An examination of IS success at the individual level. Information \& Management, 46(3), 159-166.

65. Petter, S., DeLone, W., \& McLean, E. R. (2013). Information Systems Success: The Quest for the Independent Variables. Journal of Management Information Systems, 29(4), 7-62.

66. Rai, A., Lang, S. S., \& Welker, R. B. (2002). Assessing the validity of IS success models: An empirical test and theoretical analysis. Information Systems Research, 13(1), 50-69.

67. Reix, R. (2004). Systèmes d'information et management des organisations (Vuibert). France. 
68. Reix, R., Fallery, B., Kalika, M., \& Rowe, F. (2011). Systèmes d'information et management des organisations. Vuibert.

69. Reix, R., \& Rowe, F. (2002). La recherche en systèmes d'information: de 1'histoire au concept. Faire de La Recherche En Système d'information, 1-17.

70. Roussel, P. (2000). La Motivation au Travail-Concept et Theories. Notes du Laboratoire Interdisciplinaire de recherché sur les Ressources Humaines et l'Emploi (LIRHE), Note No. 326. Toulouse: LIRHE

71. Sabherwal, R., Jeyaraj, A., \& Chowa, C. (2006). Information System Success: Individual and Organizational Determinants. Management Science, 52(12), 1849-1864.

72. Samuel, K. E., \& Ruel, S. (2013). Systèmes d'information et résilience des chaînes logistiques globales. Systèmes d'information \& management, me 18(1), 57-85.

73. Sonnentag, S., \& Frese, M. (2002). Performance concepts and performance theory. Psychological Management of Individual Performance, 23(1), 3-25.

74. Sørum, H., Andersen, K. N., \& Vatrapu, R. (2012). Public websites and human-computer interaction: an empirical study of measurement of website quality and user satisfaction. Behaviour \& Information Technology, 31(7), 697-706.

75. Tam, C., \& Oliveira, T. (2016). Understanding the impact of $\mathrm{m}-$ banking on individual performance: DeLone \& McLean and TTF perspective. Computers in Human Behavior, 61, 233-244.

76. Tam, C., \& Oliveira, T. (2017a). Literature review of mobile banking and individual performance. International Journal of Bank Marketing, 35(7), 1044-1067.

77. Tam, C., \& Oliveira, T. (2017b). Understanding mobile banking individual performance: The DeLone \& McLean model and the moderating effects of individual culture. Internet Research, 27(3), 538-562.

78. Torkzadeh, G., \& Doll, W. J. (1999). The development of a tool for measuring the perceived impact of information technology on work. Omega, 27(3), 327-339.

79. Tyndall, G., Gopal, C., Partsch, W., \& Kamauff, J. (1998). Supercharging supply chains. New ways to increase value through global operational excellence (John Wiley \& Sons). New York.

80. Urbach, N., Smolnik, S., \& Riempp, G. (2008). A methodological examination of empirical research on information systems success: 2003 to 2007. In AMCIS 2008 Proceedings (pp. 1-14). Toronto, ON, Canada A. 
81. Venkatesh, V., Morris, M. G., Davis, G. B., \& Davis, F. D. (2003). User Acceptance of Information Technology: Toward a Unified View. MIS Quarterly, 27(3), 425-478.

82. Wackermann, G., \& Corbin, E. (2005). La logistique mondiale : transport et communication. Ellipses.

83. Wagner, S. M., Grosse-Ruyken, P. T., \& Erhun, F. (2012). The link between supply chain fit and financial performance of the firm. Journal of Operations Management, 30(4), 340-353.

84. Wood, L. C., Reiners, T., \& Pahl, J. (2015). Manufacturing and logistics information systems. Encyclopedia of Information Science and Technology. Hershey, PA: IGI Global, 5136 - 5144.

85. Yatin, S. F. M., Ramli, A. A. M., Shuhaimi, H., Hashim, H., Dollah, W. A. K. W., Zaini, M. K., \& Kadir, M. R. A. (2015). Electronic Document Management System : IMalaysian Experience, 9(3), 82-89. 